\title{
Global Quasi-Minimal Residual Method for Image Restoration
}

\author{
Jun Liu, Ting-Zhu Huang, Xiao-Guang Lv, Hao Xu, and Xi-Le Zhao \\ School of Mathematical Sciences/Institute of Computational Science, University of Electronic Science and \\ Technology of China, Chengdu, Sichuan 611731, China \\ Correspondence should be addressed to Ting-Zhu Huang; tingzhuhuang@126.com
}

Received 13 August 2014; Accepted 14 October 2014

Academic Editor: Shaofan Li

Copyright (C) 2015 Jun Liu et al. This is an open access article distributed under the Creative Commons Attribution License, which permits unrestricted use, distribution, and reproduction in any medium, provided the original work is properly cited.

The global quasi-minimal residual (QMR) method is a popular iterative method for the solution of linear systems with multiple right-hand sides. In this paper, we consider the application of the global QMR method to classical ill-posed problems arising from image restoration. Since the scale of the problem is usually very large, the computations with the blurring matrix can be very expensive. In this regard, we use a Kronecker product approximation of the blurring matrix to benefit the computation. In order to reduce the disturbance of noise to the solution, the Tikhonov regularization technique is adopted to produce better approximation of the desired solution. Numerical results show that the global QMR method outperforms the classic CGLS method and the global GMRES method.

\section{Introduction}

In the area of remote sensing, materials science, medical and astronomical imaging, and so on, image restoration plays an important role in preprocessing and postprocessing the image [1]. Many image restoration tasks can be posed as problems of the form

$$
\iint_{\Omega} h(x, y ; s, t) f(s, t) d s d t=g(x, y),
$$

where the functions $f, g$ represent the original and blurred images, respectively. The kernel $h$ is a point spread function (PSF) which is a function that specifies the degree of blurring. PSFs are often classified as either spatially variant or spatially invariant $[2,3]$. For simplicity, we take into account spatially variant PSF in this paper. By means of discretization methods such as the Galerkin method or quadrature method [4], (1) can be discretized to the following linear equations:

$$
A x=\widetilde{b}, \quad A \in \mathbb{R}^{n^{2} \times n^{2}}, x, \widetilde{b} \in \mathbb{R}^{n^{2}},
$$

where $x$ is a vector representing the true image and $\widetilde{b}$ is a vector representing the blurred image, which are the discretized versions of $f$ and $g$ in (1), respectively. The matrix
$A$ is the blurring matrix constructed from the discretized version of the PSF $h$. It should be noted that the PSF is assumed to be known here. In fact, if the PSF is unknown, there are a variety of means of techniques available for estimating it $[5,6]$. In real applications, the right-hand side error-free vector $\widetilde{b}$ is not accessible. Instead, the vector

$$
b=\widetilde{b}+\eta
$$

is known, where the vector $\eta$ represents the additive noise. That is, the observed image is not only blurred but also contaminated with noise. Commonly, $\eta$ is assumed to be the white Gaussian noise, and its Euclidean vector norm is considered to be a priori but the noise vector itself is not.

In this work, we aim to obtain an approximation of the original image $x$ by computing a solution of the linear system of equations

$$
A x=b, \quad A \in \mathbb{R}^{n^{2} \times n^{2}}, x, b \in \mathbb{R}^{n^{2}} .
$$

If the observed image array has dimension $n \times n$, then $f$ and $g$ are vectors of length $n^{2}$, and $A$ is an $n^{2} \times n^{2}$ matrix. Typical values of $n$ are 256, 512, and 1024, so the dimensions of the matrix $A$ can be extremely large [7]. Then the computations with $A$ can be very expensive. 
Fortunately, the matrix $A$ has a special structure when an appropriate boundary condition is imposed. Then the computational cost of matrix-vector multiplication can be alleviated to some extent. For large-scale problems, such as image restoration problems, the direct regularization method cannot always obtain good solutions, but the iterative method is a better choice. Krylov subspace iterative methods are the most commonly used approaches that can be employed for solving (4). In [8], the authors proposed to employ the well known BiCG and QMR methods for image restoration. They also considered using a popular iterative method GMRES which was first proposed by Saad and Schultz for image restoration in [9].

Equation (4) can be replaced by new ones involving matrix equations, if the matrix $A$ can be decomposed as Kronecker products, and then the computations with $A$ can be reduced. In [10], the authors first proposed the global Krylov subspace methods to solve the matrix equations. The methods were proved to be very effective for large-scale matrix equations. Later in [11], Bouhamidi and Jbilou applied the global GMRES method to image restoration problems. Their numerical tests demonstrated that the global GMRES method was better than the GMRES method.

Due to the error in the right-hand side and the severe ill-conditioning property of the matrix $A$, the straightforward solution of (4) typically does not yield a meaningful approximation $[4,12,13]$. Therefore, instead of solving the system (4) directly, we replace it by a nearby linear system with a less ill-conditioned matrix and solve the corresponding new linear system. This replacement is commonly referred to as regularization [9]. Probably the most renowned regularization approach to overcome ill-conditioning dates back to Tikhonov and Arsenin [14].

In this paper, we consider the implementation of the global quasi-minimal residual (QMR) method for image restoration problems. The approach discussed here can be considered as an extension and a specific real application of the method introduced in [15] where the authors applied this method to solve the general Sylvester equation. This approach is motivated by the work of Bouhamidi and Jbilou in [11]. The numerical experiments show that the global QMR method is very effective compared with the global-GMRES method and the classic conjugate gradient method for least square (CGLS) problem.

The outline of this paper is as follows. In the next section, we give some notations and definitions that will be used throughout this paper. Section 3 introduces the global QMR method for image restoration problems. We present some numerical experiments to show the efficiency of the global QMR method in Section 4. Finally concluding remarks can be found in Section 5 .

\section{Preliminaries}

As shown in [7], some blurring operators (e.g., Gaussian) are separable and therefore can be factored as Kronecker product of two matrices. The computation for the solution of (4) can be reduced if the Kronecker product approximation of $A$ is employed.
Suppose that $P \in \mathbb{R}^{n \times n}$ is the discretized PSF. If the PSF is separable, that is, $P$ can be decomposed as

$$
P=\mathbf{a b}^{T}
$$

where $\mathbf{a}$ and $\mathbf{b}$ are $n \times 1$ vectors, the matrix $A$ constructed from $P$ has block structure of the form

$$
A=A_{r} \otimes A_{c}=\left(\begin{array}{cccc}
a_{11}^{r} A_{c} & a_{12}^{r} A_{c} & \cdots & a_{1 n}^{r} A_{c} \\
a_{21}^{r} A_{c} & a_{22}^{r} A_{c} & \cdots & a_{2 n}^{r} A_{c} \\
\vdots & \vdots & & \vdots \\
a_{n 1}^{r} A_{c} & a_{n 2}^{r} A_{c} & \cdots & a_{n n}^{r} A_{c}
\end{array}\right),
$$

where the matrices $A_{r}$ and $A_{c}$ have parameters a and b, respectively, with the specific structures depending on the imposed boundary condition $[6,16]$, and “ $\otimes$ " denotes Kronecker product. We refer the readers to [17] for details about the properties of Kronecker product.

If the PSF $P$ is inseparable, then the corresponding matrix $A$ is inseparable. However, we can find the Kronecker product approximation of $A$ by using SVD technique so that $A$ can be approximately decomposed as the following form:

$$
A \approx \sum_{i=1}^{r} A_{\mathbf{a}_{i}} \otimes A_{\mathbf{b}_{i}}
$$

where $P=\sum_{i=1}^{r} \mathbf{b}_{i} \mathbf{a}_{i}^{T}$ with a given integer $r \leq \operatorname{rank}(P)$. In particular, the authors in $[2,16]$ pointed out that $A_{\mathbf{a}_{1}} \otimes A_{\mathbf{b}_{1}}$ is the best (as measured by the Frobenius norm) Kronecker approximation of $A$.

According to the properties of Kronecker product, (4) can be rewritten as

$$
A_{c} X A_{r}^{T}=B
$$

where $x=\operatorname{vec}(X)$ and $b=\operatorname{vec}(B)$. Note that $z=\operatorname{vec}(Z)$ with $Z \in \mathbb{R}^{n \times n}$ is the $n^{2} \times 1$ vector obtained by stacking $n$ columns of the matrix $Z$. Define an operator $\mathscr{A}: X \in \mathbb{R}^{n \times n} \rightarrow A_{c} X A_{r}^{T}$ and $\mathscr{A}^{T}: X \in \mathbb{R}^{n \times n} \rightarrow A_{r} X A_{c}^{T}$; then (4) can be rewritten as

$$
\mathscr{A} X=B \text {. }
$$

We use the notation

$$
\mathscr{K}_{m}(\mathscr{A}, V)=\operatorname{span}\left\{V, \mathscr{A} V, \mathscr{A}^{2} V, \ldots, \mathscr{A}^{m-1} V\right\},
$$

for the global Krylov subspace of $\mathbb{R}^{n \times n}$ generated by the matrix $V \in \mathbb{R}^{n \times n}$ and the operator $\mathscr{A}$. Note that

$$
Z \in \mathscr{K}_{m}(\mathscr{A}, V) \Longleftrightarrow Z=\sum_{i=1}^{m} \alpha_{i} \mathscr{A}^{i-1} V, \quad \alpha_{i} \in \mathbb{R} .
$$

Let $X, Y \in \mathbb{R}^{n \times n}$; we define the inner matrix product $\langle X, Y\rangle_{F}=\operatorname{tr}\left(X^{T} Y\right)$, where $\operatorname{tr}(Z)$ denotes the trace of the square matrix $Z$ and $X^{T}$ the transpose of the matrix $X$. The associated norm is the Frobenius norm $\|\cdot\|_{F}$. The matrices $X, Y$ are said to be F-orthonormal if $\operatorname{tr}\left(X^{T} Y\right)=0$.

In the following, we will introduce an algorithm of the global Lanczos biorthogonal process, which has been 
(1) Given $\mathscr{A}, V_{1}$ and $W_{1}$ such that $\left\langle V_{1}, W_{1}\right\rangle_{F}=1$;

(2) Set $\beta_{1}=\delta_{1}=0$ and $V_{0}=W_{0}=O_{n \times n}$;

(3) For $i=1,2, \ldots, m$

$$
\begin{aligned}
& \alpha_{j}=\operatorname{tr}\left(W_{j}^{T} \mathscr{A} V_{j}\right) ; \\
& \widehat{V}_{j+1}=\mathscr{A} V_{j}-\alpha_{j} V_{j}-\beta_{j} V_{j-1} ; \\
& \widehat{W}_{j+1}=\mathscr{A}^{T} W_{j}-\alpha_{j} W_{j}-\delta_{j} W_{j-1} ; \\
& \delta_{j+1}=\left|\operatorname{tr}\left(\widehat{V}_{j+1}^{T} \widehat{W}_{j+1}\right)\right|^{1 / 2}, \text { if } \delta_{j+1}=0, \text { stop; } \\
& \beta_{j+1}=\operatorname{tr}\left(\widehat{V}_{j+1}^{T} \widehat{W}_{j+1}\right) / \delta_{j+1} ; \\
& V_{j+1}=\widehat{V}_{j+1} / \delta_{j+1} ; \\
& \quad W_{j+1}=\widehat{W}_{j+1} / \beta_{j+1} ; \\
& \text { End }
\end{aligned}
$$

Algorithm 1: The global Lanczos biorthogonal process.

elaborately discussed in $[15,18]$. This process is used to construct a pair of biorthogonal basis $V_{1}, V_{2}, \ldots, V_{m}$ and $W_{1}, W_{2}, \ldots, W_{m}$ of the two Krylov subspaces $\mathscr{K}_{m}\left(\mathscr{A}, V_{1}\right)$ and $\mathscr{K}_{m}\left(\mathscr{A}^{T}, W_{1}\right)$, respectively, such that

$$
\begin{aligned}
\left\langle V_{i}, W_{j}\right\rangle & =\operatorname{tr}\left(V_{i}^{T} W_{j}\right)=\delta_{i j} \\
& =\left\{\begin{array}{ll}
1, & i=j, \\
0, & i \neq j .
\end{array} \text { for } i, j=1,2, \ldots, m .\right.
\end{aligned}
$$

The construction process can be summarized as in Algorithm 1.

For convenience, we denote by $\mathscr{V}_{m}$ and $\mathscr{W}_{m}$ the $n \times n m$ block matrix, that is, $\mathscr{V}_{m}=\left[V_{1}, V_{2}, \ldots, V_{m}\right]$ and $\mathscr{W}_{m}=$ $\left[W_{1}, W_{2}, \ldots, W_{m}\right]$; two matrices are of dimension $n \times m n$. Suppose that the tridiagonal matrix $T_{m}$ is denoted by

$$
T_{m}=\left(\begin{array}{cccc}
\alpha_{1} & \beta_{2} & & \\
\delta_{2} & \alpha_{2} & \ddots & \\
& \ddots & \ddots & \beta_{m-1} \\
& & \delta_{m} & \alpha_{m}
\end{array}\right),
$$

where $\alpha_{i}, \beta_{i}$, and $\delta_{i}(i=1,2, \ldots, m)$ are the scalars defined in Algorithm 1 .

To derive the relation between $\mathscr{A} \mathscr{V}_{m}$ and $T_{m}$, we define the matrix $\widetilde{T}_{m}=\left(\begin{array}{c}T_{m} \\ \delta_{m+1} e_{m}^{T}\end{array}\right)$, where $e_{m}=(0, \ldots, 0,1)^{T} \in \mathbb{R}^{m}$.

Recall the notation $*$ in [10]:

$$
\mathscr{V}_{m} * y=\sum_{i=1}^{m} y^{i} V_{i}=\mathscr{V}_{m}\left(y \otimes I_{n}\right),
$$

where $y=\left(y_{1}, y_{2}, \ldots, y_{m}\right)^{T}$ is a vector of $\mathbb{R}^{m}, I_{n}$ is the $n \times n$ identity matrix, and

$$
\mathscr{V}_{m} * T_{m}=\left[\mathscr{V}_{m} * T_{,, 1}, \ldots, \mathscr{V}_{m} * T_{., m}\right],
$$

where $T_{., i}$ denotes the $i$ th column of the matrix $T_{m}$. Then, for $j=1,2, \ldots, m-1$, we have the following relations:

$$
\begin{gathered}
\mathscr{V}_{m} * T_{., j}=\alpha_{j} V_{j}+\delta_{j+1} V_{j+1}+\beta_{j} V_{j-1}=\mathscr{A} V_{j}, \\
\mathscr{V}_{m} * T_{., m}=\beta_{m} V_{m-1}+\alpha_{m} V_{m} \\
=\mathscr{A}_{m}+\delta_{m+1} V_{m+1} .
\end{gathered}
$$

Then we get

$$
\begin{aligned}
\mathscr{A V}_{m} & =\mathscr{V}_{m} * T_{m}+\delta_{m+1}\left[O_{n \times n}, \ldots, O_{n \times n}, V_{m+1}\right] \\
& =\mathscr{V}_{m} * T_{m}+\delta_{m+1} e_{m}^{T} V_{m+1} \\
& =\left[\mathscr{V}_{m}, V_{m+1}\right] * \widetilde{T}_{m} .
\end{aligned}
$$

That is, by the global Lanczos biorthogonal process, we can obtain

$$
\mathscr{A} \mathscr{V}_{m}=\mathscr{V}_{m+1} * \widetilde{T}_{m} .
$$

It was pointed out in [15] that the global Lanczos algorithm had significant advantages over the Arnoldi method for its fewer matrices of storage.

\section{The Global QMR Method for Image Restoration}

The quasi-minimal residual (QMR) method was first introduced by Freund and Nachtigal [19] to solve the linear equation $A x=b$. The main idea of this algorithm is to solve the reduced tridiagonal system in a least squares sense. Additionally, the QMR method uses the look-ahead technique to avoid breakdowns in the underlying Lanczos process, which makes it more robust than the BiConjugate Gradient method (BiCG) [20], and when BiCG makes no progress at all, QMR may still show slow convergence. Since the linear system is usually of large scale in applications such as image restoration, it needs enormous computation.

Fortunately, by applying the Kronecker product approximation of the matrix $A[16,17]$, the large-scale problems such as image restoration could be simplified intensively. In [10], the authors first introduced a global approach for solving matrix equations and derived the global FOM and the global GMRES methods. These methods are generalizations of the global MINRES method proposed by Saad [20]. The authors proved that these methods were effective when applied for matrix equations of large scale and low rank [21]. More recently, Wang and Gu [15] applied the global QMR method to solve the Sylvester equations. In this work, we will focus on the global QMR method for image restoration.

Suppose that the operator $\mathscr{A}=A_{r} \otimes A_{c}$ is a good approximation of $A, x=\operatorname{vec}(X)$ and $b=\operatorname{vec}(B)$. In the following, we give details of the global QMR method for image restoration. Let $X_{0} \in \mathbb{R}^{n \times n}$ be the initial solution of (9) and let $R_{0}=B-\mathscr{A} X_{0}$ be the corresponding residual. Usually we set the black image to be the initialization. By using Algorithm 1 for (9), the iterate $X_{m}$ at step $m$ satisfies that

$$
X_{m}-X_{0} \in \mathscr{K}_{m}\left(\mathscr{A}, R_{0}\right) .
$$

Define $V_{1}=R_{0} / \rho, \rho=\left\|R_{0}\right\|_{F}$ and $W_{1}$ such that, $\left\langle V_{1}, W_{1}\right\rangle_{F}=1$. Suppose that the matrix Krylov subspaces $\mathscr{K}_{m}\left(\mathscr{A}, R_{0}\right)$ and $\mathscr{K}_{m}\left(\mathscr{A}^{T}, W_{1}\right)$ are generated by the sets of matrices $\left\{V_{1}, V_{2}, \ldots, V_{m}\right\}$ and $\left\{W_{1}, W_{2}, \ldots, W_{m}\right\}$ constructed by Algorithm 1. Then according to (19), we can obtain an approximate solution of (9):

$$
X_{m}=X_{0}+\mathscr{V}_{m} * \mathbf{y}_{m},
$$


where $\mathbf{y}_{m}=\left(y_{1}, y_{2}, \ldots, y_{m}\right)^{T}$. Consequently, we can get the associated residual matrix

$$
\begin{aligned}
R_{m} & =B-\mathscr{A} X_{m} \\
& =B-\mathscr{A}\left(X_{0}+\mathscr{V}_{m} * \mathbf{y}_{m}\right) \\
& =R_{0}-\mathscr{A} \mathscr{V}_{m} * \mathbf{y}_{m} \\
& =\rho V_{1}-\mathscr{V}_{m+1} * \widetilde{T}_{m} \mathbf{y}_{m} \\
& =\mathscr{V}_{m+1} *\left(\rho e_{1}-\widetilde{T}_{m} \mathbf{y}_{m}\right),
\end{aligned}
$$

where $e_{1}=(1,0, \ldots, 0)^{T}$. Hence, the norm of the residual matrix is

$$
\left\|R_{m}\right\|_{F}=\left\|\mathscr{V}_{m+1} *\left(\rho e_{1}-\widetilde{T}_{m} \mathbf{y}_{m}\right)\right\|_{F} .
$$

An approximate solution of (9) can be obtained by computing the minimizer from (22) with respect to $\mathbf{y}_{m}$. Generally, the $V_{i}$ 's obtained by Algorithm 1 are not F-orthonormal. However, as shown in [20], it is still reasonable to obtain that

$$
\widehat{\mathbf{y}}_{m}=\arg \min _{y_{m} \in \mathbb{R}^{m}}\left\|\rho e_{1}-\widetilde{T}_{m} \mathbf{y}_{m}\right\|_{F} .
$$

What has been shown above is the key idea of the global QMR method; hence the approximated solution by the global QMR method can be given as

$$
X_{m}=X_{0}+\mathscr{V}_{m} * \widehat{\mathbf{y}}_{m}
$$

where $\widehat{\mathbf{y}}_{m}=\arg \min _{y_{m} \in \mathbb{R}^{m}}\left\|\rho e_{1}-\widetilde{T}_{m} \mathbf{y}_{m}\right\|_{F}$. We refer the readers to see $[15,20]$ for the details on how to compute $\widehat{\mathbf{y}}_{m}$.

To sum up, the algorithm for obtaining the approximate solution of (9) arising from image restoration can be described as in Algorithm 2.

We note that the discrepancy principle can be used as the stopping criterion in Algorithm 2; that is, the computations will be terminated if the associated residual error corresponds to the approximate solution $X_{m}$

$$
\left\|\mathscr{A} X_{m}-B\right\| \leq \mu \epsilon, \quad\left\|\mathscr{A} X_{m-1}-B\right\|>\mu \epsilon,
$$

where $\epsilon$ is the noise's Frobenius Norm which is supposed to be a priori and $\mu \geq 1$ is a fixed constant. For details on the discrepancy principle, we refer to [22] and references therein for more details.

Since the matrix $A$ is usually ill-conditioned, the solution is sensitive to the noise in the observed image. In the following, in order to improve the accuracy of the solution, we consider combining the global QMR method with Tikhonov regularization technique. Motivated by the work in [11], we can obtain the following algorithm which is named as the global Tik-QMR method.

In Algorithm 3, the regularization parameter $\lambda$ can be determined by the L-curve criterion or the GCV method. We choose the latter here. Note that the regularization step in our work is different from the work in [11], since we adopt the regularization after Lanczos process while the authors in [11] used the regularization before the Lanczos process. Then our method needs fewer computations than theirs.
(1) Given $\mathscr{A}$, and $B, X_{0}$;

(2) Set $R_{0}=\widehat{B}-\mathscr{A} X_{0}, \rho=\left\|R_{0}\right\|_{F}, W_{1}=V_{1}=R_{0} / \rho$;

(3) For $m=1,2, \ldots$

Compute $\widetilde{T}_{m}, V_{m}, W_{m}$ using Algorithm 1;

$\widehat{\mathbf{y}}_{m}=\arg \min _{\mathbf{y}_{m} \in \mathbb{R}^{m}}\left\|\rho e_{1}-\widetilde{T}_{m} \mathbf{y}_{m}\right\|_{2}$;

$X_{m}=X_{0}+\mathscr{V}_{m} * \widehat{\mathbf{y}}_{m}$

End

Algorithm 2: The global QMR method for image restoration.

(1) Given $\mathscr{A}, \widehat{B}, X_{0}, \mu$ and $\epsilon$;

(2) Set $R_{0}=\widehat{B}-\mathscr{A} X_{0}, \rho=\left\|R_{0}\right\|_{F}, W_{1}=V_{1}=R_{0} / \rho$;

(3) For $m=1,2, \ldots$

Compute $\widetilde{T}_{m}, V_{m}, W_{m}$ using Algorithm 1;

$\widehat{\mathbf{y}}_{m}=\arg \min _{\mathbf{y}_{m} \in \mathbb{R}^{m}}\left\|\rho e_{1}-\widetilde{T}_{m} \mathbf{y}_{m}\right\|_{2}^{2}+\lambda^{2}\left\|\mathbf{y}_{m}\right\|_{2}^{2}$;

$X_{m}=X_{0}+\mathscr{V}_{m} * \widehat{\mathbf{y}}_{m}$;

$R_{m}=\mathscr{V}_{m+1} *\left(\rho e_{1}-\widetilde{T}_{m} \mathbf{y}_{m}\right)$;

if $\left\|R_{m}\right\|_{F}$ satisfies the discrepancy principle, stop else

End

$$
X_{0}=X_{m}, \rho=\left\|R_{m}\right\|_{F}, V_{1}=R_{m} / \rho
$$

Algorithm 3: The global Tik-QMR method for image restoration.

\section{Numerical Experiments}

In this section, we report some numerical examples to illustrate the performance of the global QMR method for image restoration problems. The results show that the quality of images restored by the global QMR method is better than those obtained by other methods of the same kind, such as the classic CGLS method [23] and the global GMRES method proposed by Jbilou et al. [10]. The experiments are carried out in Matlab 7.0 on a PC equipped with a $2.93 \mathrm{GHz}$ Intel Core Duo CPU, with 2 GB of RAM, under Microsoft Windows XP.

Example 1. Our first example is to show the practical efficiency of the global QMR method. The original image $X$ of size $256 \times 256$ is shown in Figure 1(a), which can be obtained from the Telescope Science Institute, and intended to simulate a star cluster image taken by the Hubble space telescope before its defective mirror was replaced [24]. Let $X$ denote the exact star cluster. The PSF used in this example is the socalled Moffat function [6]. The PSF is given by

$$
P_{i j}= \begin{cases}\left((i-16)^{2}+\left(\frac{j-16}{2}\right)^{2}\right)^{-5} & \text { if } 1 \leq i, j \leq 30, \\ 0, & \text { otherwise. }\end{cases}
$$

This PSF $P$ is nonsymmetric and unseparable, so the blurring matrix $A$ constructed from $P$ is nonsymmetric and unseparable. If the zero boundary condition is imposed, the matrix $A$ can be represented as the Kronecker product approximation of Toeplitz matrices $A_{r}$ and $A_{c}$; that is, $A=$ $A_{r} \otimes A_{c}$. We add $1 \%$ white Gaussian noise to the blurred image 
TABLE 1: Numerical results for the experiments, in terms of PSNR (dB) and CPU time (second).

\begin{tabular}{|c|c|c|c|c|}
\hline Test problem & Method & restored PSNR & CPU time & observed PSNR \\
\hline starcluster & global QMR & 41.16 & $0.25 \mathrm{~s}$ & 30.82 \\
\hline \multirow{2}{*}{ MRI } & global QMR & 20.61 & $0.156 \mathrm{~s}$ & \multirow{2}{*}{17.95} \\
\hline & global Tik-QMR & 22.16 & $0.344 \mathrm{~s}$ & \\
\hline \multirow{2}{*}{ Indian man } & global Tik-QMR & 25.69 & $8.078 \mathrm{~s}$ & \multirow{2}{*}{23.51} \\
\hline & CGLS & 25.05 & $1.7 \mathrm{~s}$ & \\
\hline \multirow{2}{*}{ bridge } & global Tik-QMR & 19.91 & $49.42 \mathrm{~s}$ & \multirow{2}{*}{15.66} \\
\hline & global GMRES & 20.05 & $67.95 \mathrm{~s}$ & \\
\hline
\end{tabular}

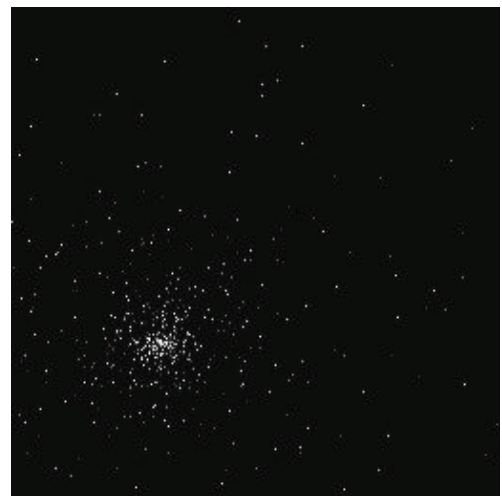

(a) Exact image

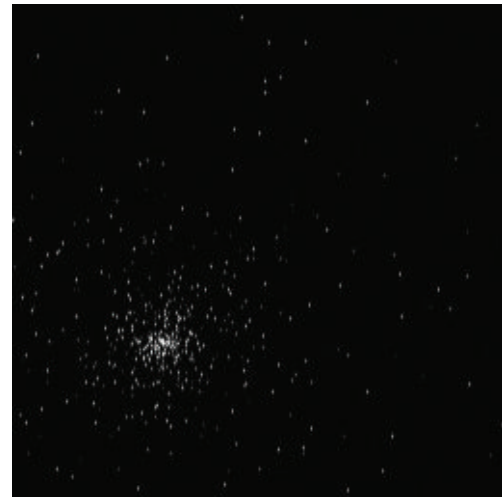

(b) Observed image

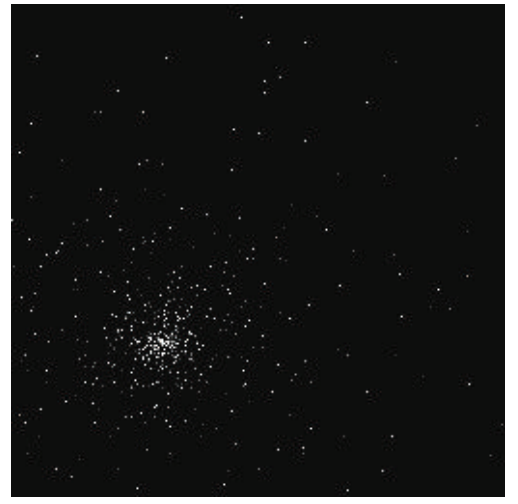

(c) Restored image

FIGURE 1: Example 1: (a) original image. (b) Observed image contaminated with blur and noise. (c) Image restored by the global QMR method.

to simulate the observed image (Figure 1(b)). The PSNR of the observed image is $30.82 \mathrm{~dB}$. We set the parameter $\mu=$ 1.05 for the discrepancy principle. Using the global QMR method, we obtained the estimated image after 4 iterations when the discrepancy principle of its associated residual is satisfied. The numerical results in terms of PSNR are reported in Table 1. From the table, we see that the PSNR of the restored image by the global QMR method is $41.16 \mathrm{~dB}$ and the consumed CPU time is $0.25 \mathrm{~s}$. The restored image is shown in Figure 1(c).

Example 2. In order to suppress the sensitivity of solution to noise, we employ Tikhonov regularization technique to get a more accurate solution. In this example, we compared the performance of the global Tik-QMR method and the global QMR method. We consider the problem of restoring the image of the MRI data from Matlab (Figure 2(a)). The data size is $128 \times 128$. The blurred and noisy image is shown in Figure 2(b). The PSF for blurring in this test is the truncated separable Gaussian function, and the variance of the Gaussian blur is 3 and $1 \%$ white Gaussian noise is added.

The restored images obtained by the global QMR method and the global Tik-QMR method are shown in Figure 3, respectively. From Figure 3, it is easy to see that the image restored by the global Tik-QMR method has higher visual quality than that by the global QMR method. The numerical results are shown in Table 1 , and it is not difficult to see that the global Tik-QMR method outperforms the global QMR method.

Example 3. The third example consists in restoring the image of $512 \times 512$ "Indian man" degraded by the Gaussian blur and $0.1 \%$ additive noise. The true image and degraded image are shown in Figure 4. We compare the global Tik-QMR method with the classic CGLS. The PSNR of the restored images by the two methods and computational CPU time are given in Table 1. The restored images are shown in Figure 5.

From Figure 5 and Table 1, we see that the global TikQMR method is quite competitive with the CGLS method.

Example 4. In the last experiment, the $256 \times 256$ bridge image has been contaminated by a nonsymmetric wavefront blur [25] and $0.1 \%$ additive noise. The true image, the wavefront PSF, and the degraded image are shown in Figure 6. The PSF is also unseparable. Then the corresponding blurring matrix $A$ is approximated by the Kronecker product of two small matrices. We compared the behavior of the global TikQMR method and the global GMRES method [11] in this experiment.

The numerical results are given in Table 1 . From the table, we see that the PSNR of the restored image by the global GMRES method is slightly higher than the global TikQMR method, but the CPU time by using the global TikQMR method is much less than the global GMRES method. 


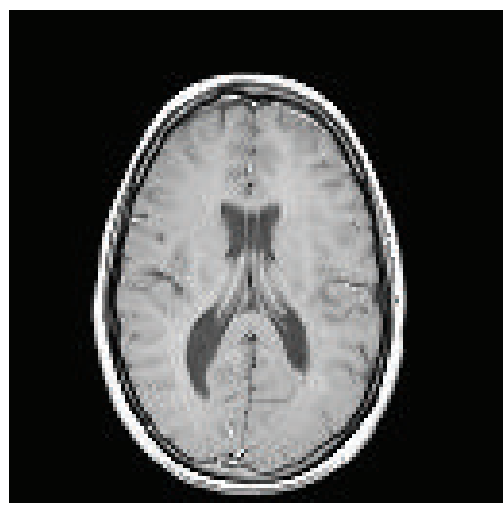

(a) Exact image

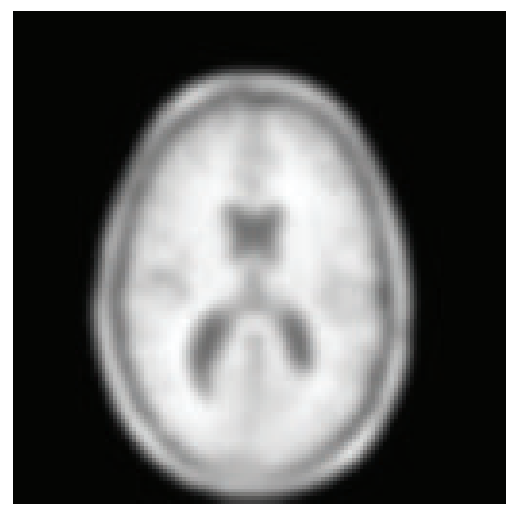

(b) Observed image

Figure 2: Example 2: exact and observed images.

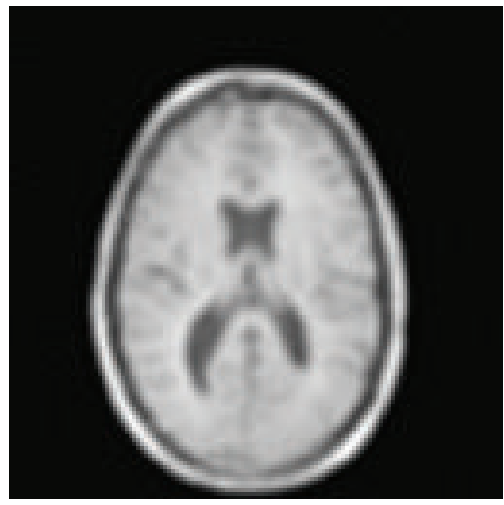

(a) Global QMR image

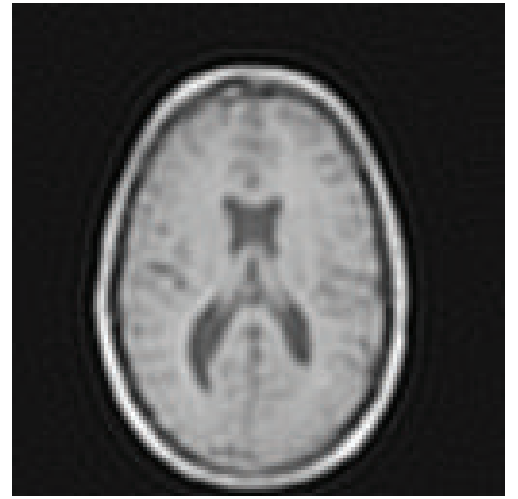

(b) Global Tik-QMR image

FIgURE 3: Example 2: restored images by the global QMR method and the global Tik-QMR method.

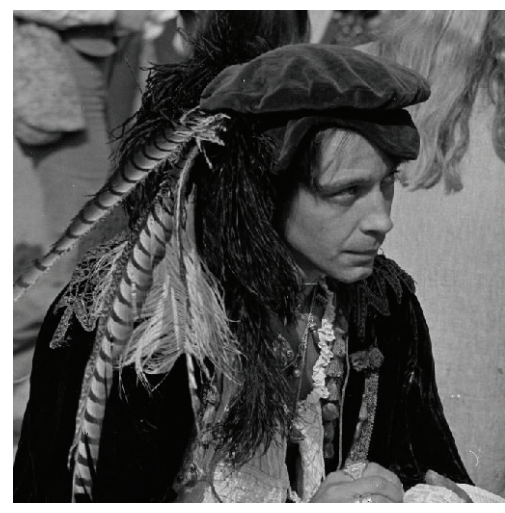

(a) Exact image

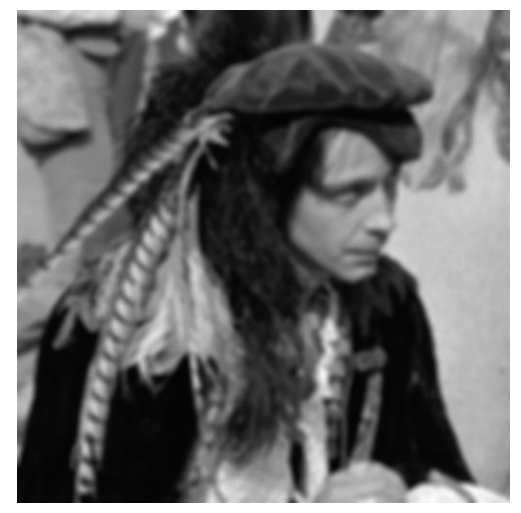

(b) Observed image

FIgURE 4: Example 3: exact and observed images.

The visual quality of restored images is very close. The restored images by using the two methods are displayed in Figure 7.

At the end of this section, a general comment about the presented numerical experiments is worth mentioning. The first example illustrates efficiency of the proposed method for image restoration problems. In general, the global Tik-QMR method behaves better than the classic CGLS method and the global GMRES method.

\section{Conclusion}

In [10], Jbilou et al. first introduced the global methods. In this paper, we take the advantage of the global QMR method 


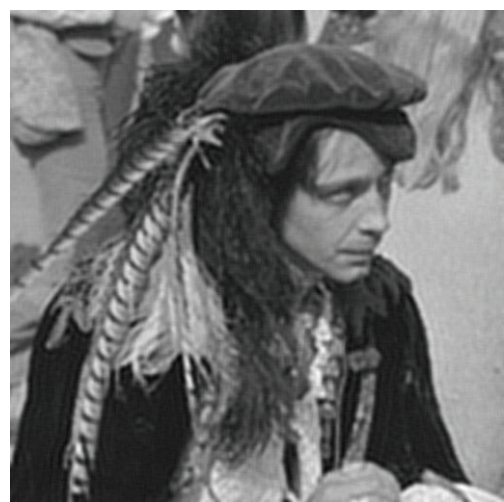

(a) Global Tik-QMR image

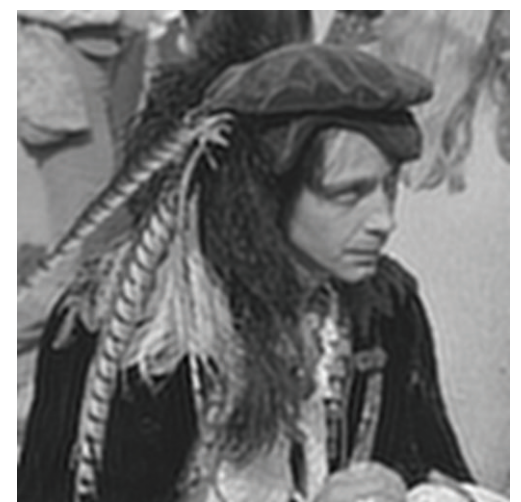

(b) CGLS image

FIgURE 5: Example 3: exact and observed images.

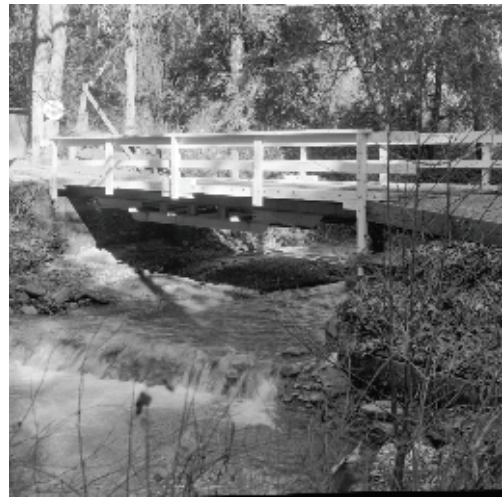

(a) Exact image

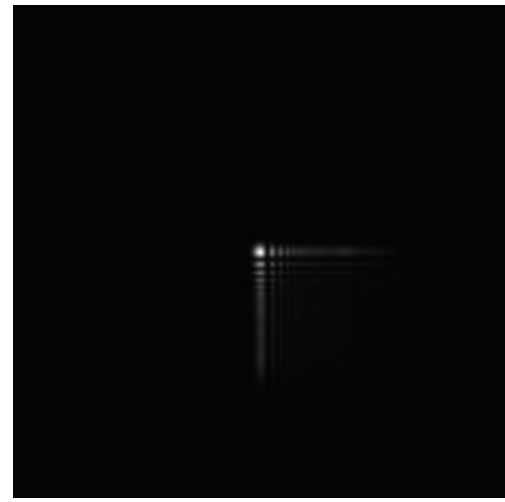

(b) Wavefront PSF

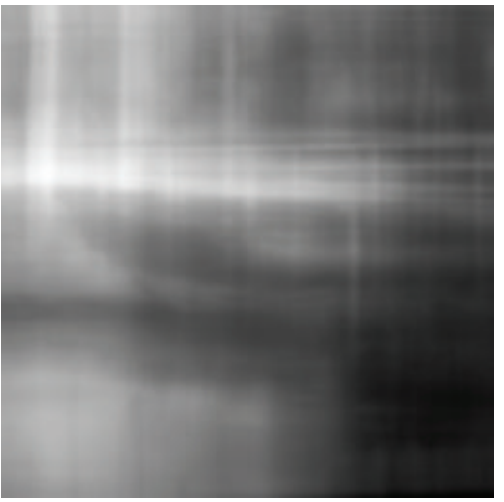

(c) Observed image

FIGURE 6: Example 4: exact image, wavefront PSF, and observed image.

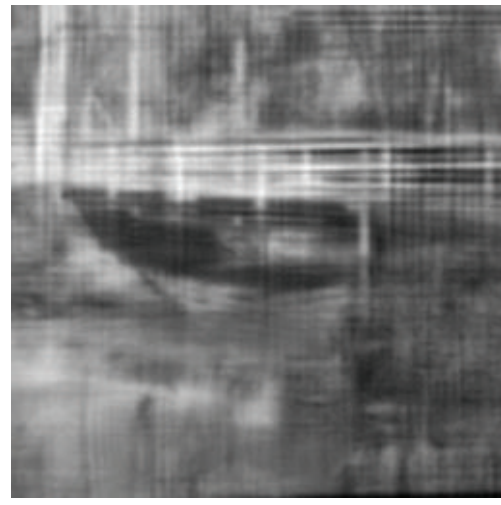

(a) Global GMRES image

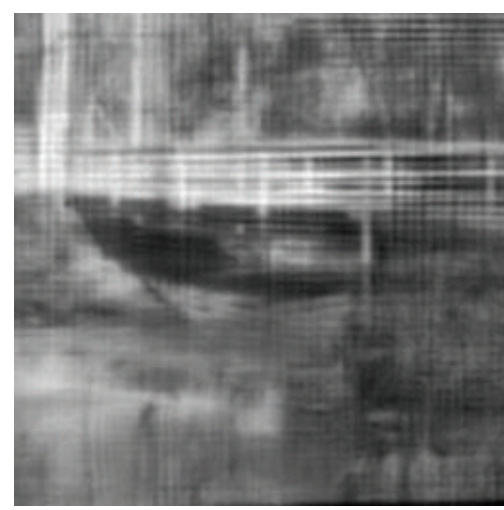

(b) Global Tik-QMR image

FIGURE 7: Example 4: restored images using the global GMRES method and the global Tik-QMR method. 
for image restoration and compare it with other popular methods. Numerical results show that the global QMR method is very efficient and is competitive with the classic CGLS method and the global GMRES method in [11]. In addition, when combining with the Tikhonov regularization, the global QMR method can behave much better.

\section{Conflict of Interests}

The authors declare that there is no conflict of interests regarding the publication of this paper.

\section{Acknowledgments}

This work is supported by NSFC (61370147, 61170311), 973 Program (2013CB329404), and Sichuan Province Sci. \& Tech. Research Project (2012GZX0080).

\section{References}

[1] M. R. Banham and A. K. Katsaggelos, "Digital image restoration," IEEE Signal Processing Magazine, vol. 14, no. 2, pp. 24-41, 1997.

[2] J.-G. Nagy, M.-K. Ng, and L. Perrone, "Kronecker product approximations for image restoration with reflexive boundary conditions," SIAM Journal on Matrix Analysis and Applications, vol. 25, no. 3, pp. 829-841, 2003.

[3] J. G. Nagy, K. Palmer, and L. Perrone, "Iterative methods for image deblurring: a Matlab object-oriented approach," Numerical Algorithms, vol. 36, no. 1, pp. 73-93, 2004.

[4] P. C. Hansen, Rank-Deficient and Discrete Ill-Posed Problems, SIAM Monographs on Mathematical Modeling and Computation, Society for Industrial and Applied Mathematics (SIAM), Philadelphia, Pa, USA, 1997.

[5] K. T. Lay and A. K. Katsaggelos, "Identification and restoration based on the expectation-maximization algorithm," Optical Engineering, vol. 29, no. 5, pp. 436-445, 1990.

[6] P. C. Hansen, J. G. Nagy, and D. O'Leary, Deblurring Images: Matrices, Spectra, and Filtering, SIAM, Philadelphia, Pa, USA, 2006.

[7] J. Kamm and J. G. Nagy, "Kronecker product and SVD approximations in image restoration," Linear Algebra and Its Applications, vol. 284, no. 1-3, pp. 177-192, 1998.

[8] D. Calvetti, B. Lewis, and L. Reichel, "Krylov subspace iterative methods for nonsymmetric discrete ill-posed problems in image restoration," in Advanced Signal Processing Algorithms, Architectures, and Implementations XI, vol. 4474 of Proceedings of SPIE, pp. 224-233, San Diego, Calif, USA, 2001.

[9] D. Calvetti, B. Lewis, and L. Reichel, "GMRES, L-curves, and discrete ill-posed problems," BIT. Numerical Mathematics, vol. 42, no. 1, pp. 44-65, 2002.

[10] K. Jbilou, A. Messaoudi, and H. Sadok, "Global FOM and GMRES algorithms for matrix equations," Applied Numerical Mathematics, vol. 31, no. 1, pp. 49-63, 1999.

[11] A. Bouhamidi and K. Jbilou, "Sylvester Tikhonov-regularization methods in image restoration," Journal of Computational and Applied Mathematics, vol. 206, no. 1, pp. 86-98, 2007.

[12] P.-C. Hansen, Discrete Inverse Problems: Insight and Algorithms, SIAM, Philadelphia, Pa, USA, 2010.
[13] X.-G. Lv, T.-Z. Huang, Z.-B. Xu, and X.-L. Zhao, "Kronecker product approximations for image restoration with wholesample symmetric boundary conditions," Information Sciences, vol. 186, pp. 150-163, 2012.

[14] A. Tikhonov and V. Arsenin, Solution of Ill-Poised Problems, V. H. Winston \& Sons, Washington, DC, USA, 1977.

[15] Y. Wang and G.-D. Gu, "Global quasi-minimal residual method for the Sylvester equations," Journal of Shanghai University (English Edition), vol. 11, no. 1, pp. 52-57, 2007.

[16] J. G. Nagy and L. Perrone, "Kronecker products in image restoration," in 13th Advanced Signal Processing Algorithms, Architectures, and Implementations, vol. 5205 of Proceedings of SPIE, pp. 369-379, San Diego, Calif, USA, August 2003.

[17] C.-F. van Loan and N. P. Pitsianis, "Approxiation with Kronecker product," in Linear Algebra for Large Scale and Real Time Applications, M. S. Moonen and G. H. Golub, Eds., pp. 293-314, Kluwer Publications, 1993.

[18] K. Jbilou, H. Sadok, and A. Tinzefte, "Oblique projection methods for linear systems with multiple right-hand sides," Electronic Transactions on Numerical Analysis, vol. 20, pp. 119138, 2005.

[19] R. W. Freund and N. M. Nachtigal, "QMR: a quasi-minimal residual method for non-Hermitian linear systems," Numerische Mathematik, vol. 60, no. 3, pp. 315-339, 1991.

[20] Y. Saad, Iterative Methods for Sparse Linear Systems, Philadelphia, Pa, USA, 2nd edition, 2003.

[21] Y.-Q. Lin, "Implicitly restarted global FOM and GMRES for nonsymmetric matrix equations and Sylvester equations," Applied Mathematics and Computation, vol. 167, no. 2, pp. 10041025, 2005.

[22] D. Calvetti, B. Lewis, and L. Reichel, "On the regularizing properties of the GMRES method," Numerische Mathematik, vol. 91, no. 4, pp. 605-625, 2002.

[23] M. Hanke, Conjugate Gradient Type Methods for Ill-Posed Problems, Longman Scientific \& Technical, Essex, UK, 1995.

[24] D. Calvetti, G. Landi, L. Reichel, and F. Sgallari, "Non-negativity and iterative methods for ill-posed problems," Inverse Problems, vol. 20, no. 6, pp. 1747-1758, 2004.

[25] E.-R. Dowski and W.-T. Cathey, "Extended depth of field through wave-front coding," Applied Optics, vol. 34, no. 11, pp. 1859-1866, 1995. 


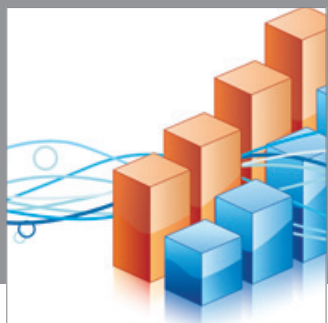

Advances in

Operations Research

mansans

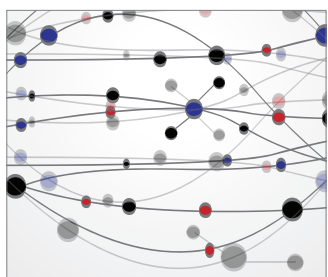

The Scientific World Journal
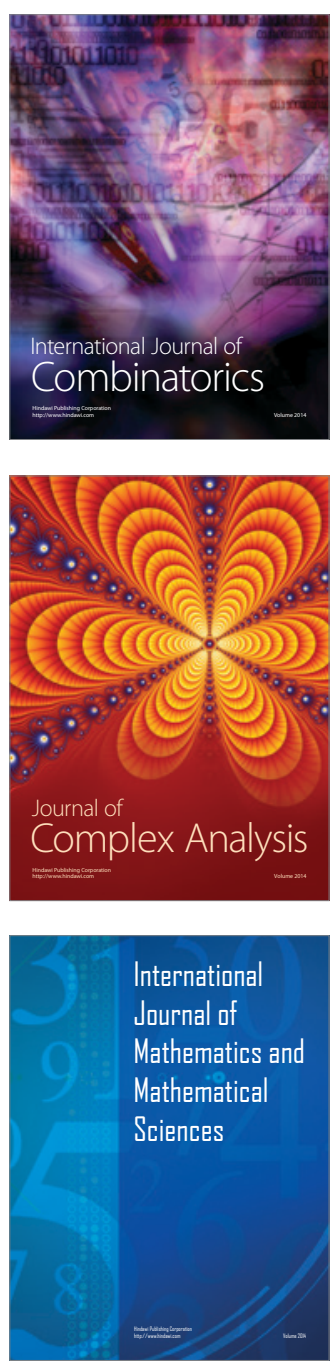
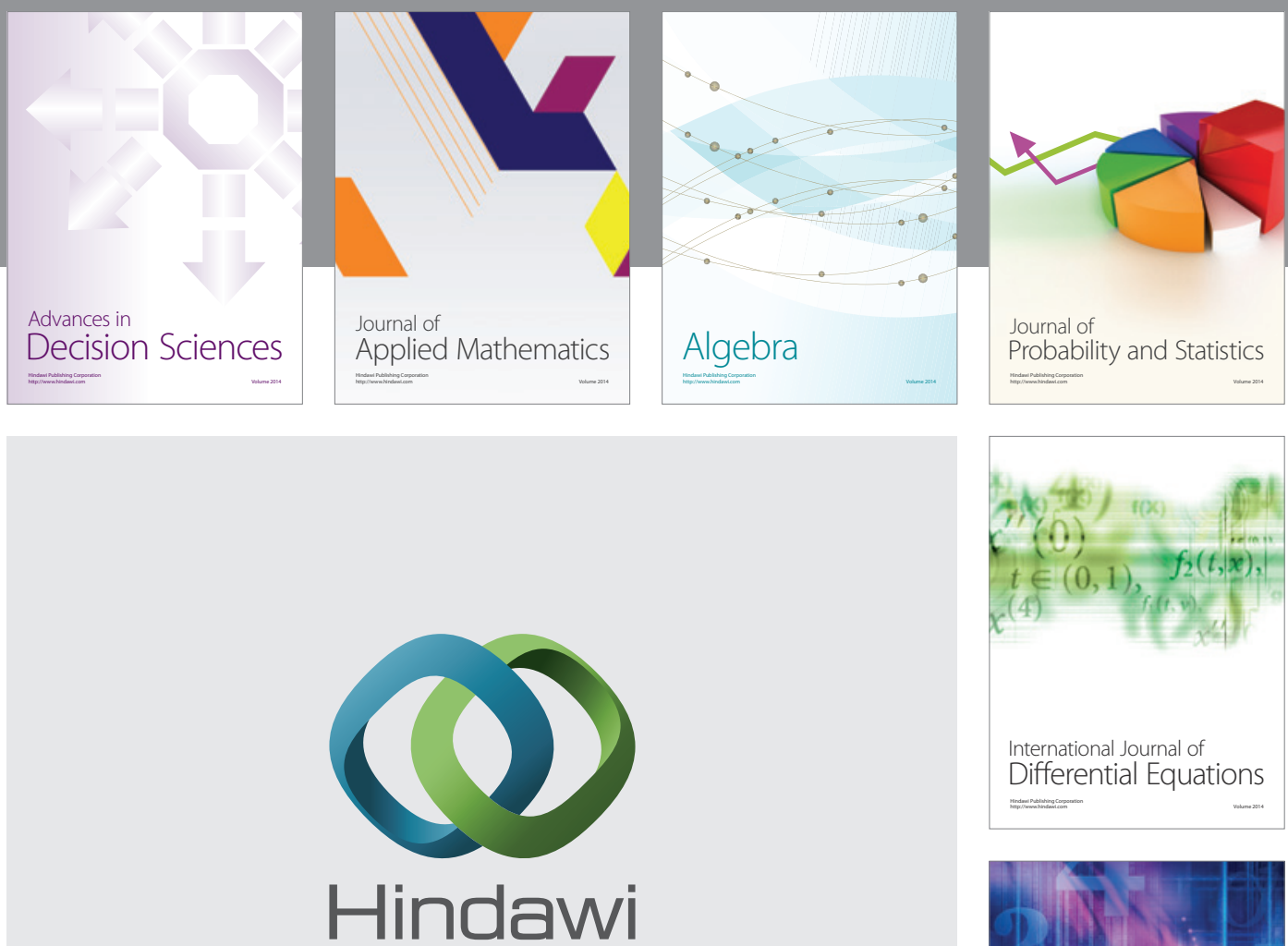

Submit your manuscripts at http://www.hindawi.com
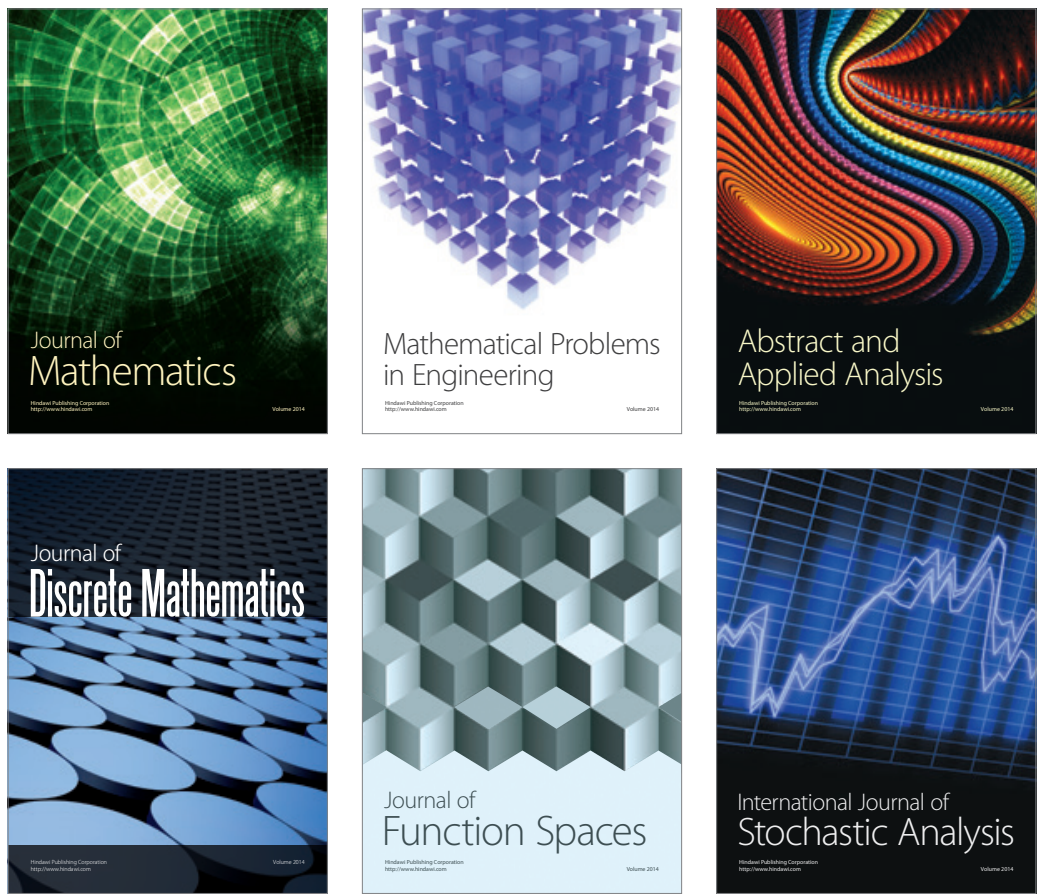

Journal of

Function Spaces

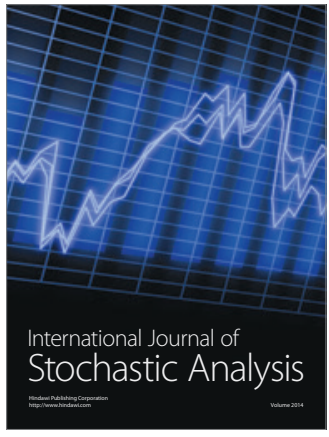

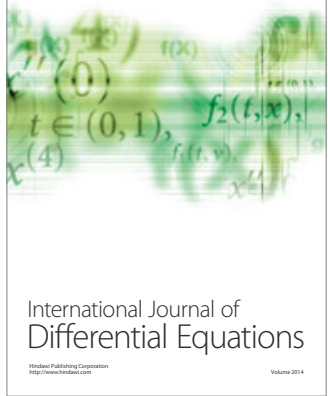
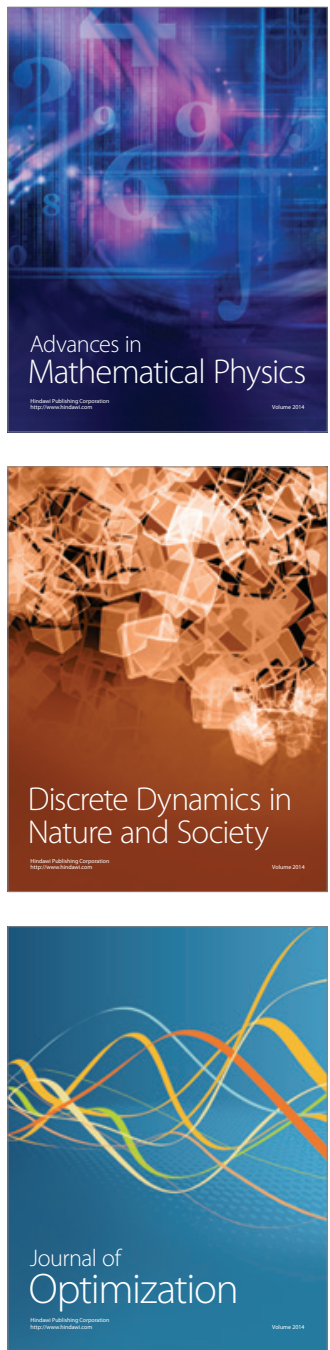